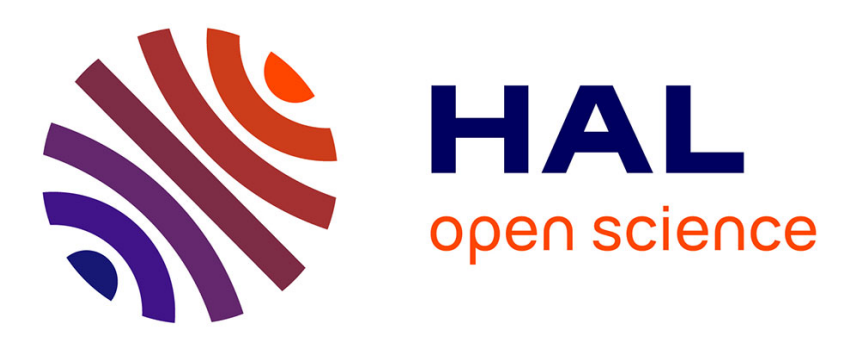

\title{
Solving self-mixing equations for arbitrary feedback levels: a concise algorithm
}

Russell Kliese, Thomas Taimre, A. Ashrif A. Bakar, Yah Leng Lim, Karl Bertling, Milan Nikolić, Julien Perchoux, Thierry Bosch, Aleksandar D. Rakić

\section{- To cite this version:}

Russell Kliese, Thomas Taimre, A. Ashrif A. Bakar, Yah Leng Lim, Karl Bertling, et al.. Solving self-mixing equations for arbitrary feedback levels: a concise algorithm. Applied optics, 2014, 53 (17), pp.3723-3736. 10.1364/AO.53.003723 . hal-01091363

\section{HAL Id: hal-01091363 https://hal.science/hal-01091363}

Submitted on 5 Dec 2014

HAL is a multi-disciplinary open access archive for the deposit and dissemination of scientific research documents, whether they are published or not. The documents may come from teaching and research institutions in France or abroad, or from public or private research centers.
L'archive ouverte pluridisciplinaire HAL, est destinée au dépôt et à la diffusion de documents scientifiques de niveau recherche, publiés ou non, émanant des établissements d'enseignement et de recherche français ou étrangers, des laboratoires publics ou privés. 


\title{
Solving self-mixing equations for arbitrary feedback levels: a concise algorithm
}

\author{
Russell Kliese, ${ }^{1}$ Thomas Taimre, ${ }^{2}$ A. Ashrif A. Bakar, ${ }^{3}$ Yah Leng Lim, ${ }^{4}$ Karl Bertling, ${ }^{4}$ \\ Milan Nikolić, ${ }^{4}$ Julien Perchoux, ${ }^{5,6}$ Thierry Bosch, ${ }^{5,6}$ and Aleksandar D. Rakić ${ }^{4, *}$ \\ 'TOPTICA Photonics AG, Lochhamer Schlag 19, 82166 Gräfelfing, Germany \\ ${ }^{2}$ School of Mathematics and Physics, The University of Queensland, Brisbane, QLD 4072, Australia \\ ${ }^{3}$ Department of Electrical, Electronic and Systems Engineering, Universiti Kebangsaan Malaysia, \\ Bangi, Selangor 43600, Malaysia \\ ${ }^{4}$ School of Information Technology and Electrical Engineering, The University of Queensland, \\ Brisbane, QLD 4072, Australia \\ ${ }^{5}$ CNRS, LAAS, F-31400 Toulouse, France \\ ${ }^{6}$ Univ de Toulouse, INP, LAAS, F-31400 Toulouse, France \\ ${ }^{*}$ Corresponding author: rakic @itee.uq.edu.au
}

Received 26 November 2013; revised 28 April 2014; accepted 28 April 2014; posted 1 May 2014 (Doc. ID 201983); published 9 June 2014

\begin{abstract}
Self-mixing laser sensors show promise for a wide range of sensing applications, including displacement, velocimetry, and fluid flow measurements. Several techniques have been developed to simulate selfmixing signals; however, a complete and succinct process for synthesizing self-mixing signals has so far been absent in the open literature. This article provides a systematic numerical approach for the analysis of self-mixing sensors using the steady-state solution to the Lang and Kobayashi model. Examples are given to show how this method can be used to synthesize self-mixing signals for arbitrary feedback levels and for displacement, distance, and velocity measurement. We examine these applications with a deterministic stimulus and discuss the velocity measurement of a rough surface, which necessitates the inclusion of a random stimulus. () 2014 Optical Society of America

OCIS codes: (280.3420) Laser sensors; (140.3430) Laser theory.

http://dx.doi.org/10.1364/AO.53.003723
\end{abstract}

\section{Introduction}

Semiconductor lasers are sensitive to feedback effects, which occur when a portion of the emitted light re-enters the active laser cavity $[\underline{1}, \underline{2}]$. This feedback sensitivity is often considered a nuisance, and various methods are used to reduce its effect in many applications. Techniques to improve feedback immunity include surface relief gratings [3], increased cavity mirror reflectivity $[\underline{4}, \underline{5}]$, and optical isolators. However, optical feedback can be used to create an interferometric sensor where the laser is both the

$1559-128 \mathrm{X} / 14 / 173723-14 \$ 15.00 / 0$

(C) 2014 Optical Society of America source and the detector. This "self-mixing" laser sensor has been demonstrated in a wide variety of sensing applications including absolute distance [6], velocity [7,8], and fluid flow [9]. The self-mixing effect is also remarkably universal and has been observed in gas lasers [10-12], quantum cascade lasers [13], interband cascade lasers [14], vertical-cavity surfaceemitting lasers (VCSELS) [15], solid-state lasers $[16,17]$, and fiber ring lasers $[18,19]$.

The self-mixing laser sensor can be modeled using laser rate equations. The rate equation model of a semiconductor laser under feedback was originally developed by Lang and Kobayashi [20] and is acknowledged to provide a realistic physical model. While the Lang and Kobayashi model can be used 
directly, it can be computationally expensive. Furthermore, the model can be simplified under temporal steady-state conditions. Therefore it is of interest to identify when the assumption of steady-state operation is justified. The Lang and Kobayashi model can be solved under steady-state conditions provided the temporal changes in the stimulus are slow compared with characteristic times associated with the system, or, in other words, the stimulus frequency has to be much lower than the natural frequency of the system-the laser relaxation frequency. Furthermore, for a laser under feedback, the laser dynamics also will be affected by the length of the external cavity, and in this case the frequency of the stimulus needs to be much smaller than the natural frequency of the external cavity resonator [21]. (A typical near infrared VCSEL has a relaxation frequency on the order of $1 \mathrm{GHz}$ with a natural cavity frequency, for a $1 \mathrm{~m}$ external cavity, on the order of $100 \mathrm{MHz}$.) These conditions are easily satisfied in many cases, even for the relatively high target velocities that have been sensed from moving cars [22].

Analysis of the Lang and Kobayashi model also provides insight into different operation regimes that depend on the level of feedback and the optical round-trip phase [23]. At high levels of feedback and/or long external path lengths, the laser can become unstable leading to coherence collapse $[4,24]$ and chaotic behavior [25-28]. In this article, we restrict ourselves to the stable regions that are most useful for sensing applications.

The Lang and Kobayashi model in the temporal steady state condition can be reduced to a single transcendental equation [29]. The same equation is also obtained from simple optical considerations of a three mirror Fabry-Perot cavity, without reference to the laser structure [30]. The mathematical equation obtained from both the steady-state rate equation model and the three-mirror model describes the phase condition for a laser under feedback, commonly referred to as the excess-phase equation. This model only considers single-mode operation and is applicable to intrinsically single-mode lasers such as distributed feedback lasers and some VCSELs. However, under some operating conditions, multimode VCSELs $[31,32]$ and in-plane cleaved faceted lasers also perform well as self-mixing sensors. Self-mixing models, which include multimodal effects, have been developed [33-35] but are beyond the scope of this article.

Numerical solution of this equation provides important insight into the way a self-mixing sensor functions. The excess-phase equation has been used to estimate semiconductor laser parameters under weak feedback [36] to improve the accuracy of a self-mixing sensor by utilizing fringe loss information (predicted by the model as the level of feedback increases) [37] and to compensate for thermal effects in laser range-finding applications [38].

Lasers under feedback exhibit complicated phenomena, including hysteresis effects and the presence of multiple possible laser oscillation modes, some of which are unstable [28]. The excess-phase equation captures this complex behavior, despite its simple form. Because of this, a series of considered steps must be followed to obtain physically meaningful solutions from the excess-phase equation. This complex behavior, including fringe loss, has been correctly captured by Plantier's behavioral model [39]. However, a complete and succinct process for direct numerical solution of the excess-phase equation has so far been absent in the open literature.

In this paper, we present a complete numerical procedure for solving the excess-phase equation for all feedback regimes and for arbitrary stimulus. Such numerical solution is indispensable for predicting the behavior of the self-mixing sensing system operating under different conditions. To this end, we describe how this straightforward method is used to synthesize self-mixing signals by focussing on three applications: displacement, absolute distance, and velocity measurement.

The paper is organized as follows. In Section $\underline{2}$, the relevant self-mixing theory and equations are presented. The process for solving the excess-phase equation is given in Section 3 . This is followed by a discussion of how the process can be applied to model a self-mixing displacement, absolute distance, and velocity sensor in Section 4 . The final section, Section $\underline{5}$, contains concluding remarks.

\section{Theory}

The self-mixing model developed here is based on the work by Petermann [30]. We do not repeat Petermann's derivations but start from the key equations in his work. We then provide a description of important considerations and relevant additional equations.

Petermann's model extends the conventional two-mirror laser model to a three-mirror model. The two-mirror laser model is based on satisfying two conditions: the optical field in the laser has the same phase after one round-trip in the laser cavity, and the round-trip optical gain is unity [40]. The laser phase condition in the three-mirror model is modified to include both the effects of the two laser facet mirrors and the effect of feedback from an external target that forms the third mirror. The emission frequency of the free-running laser, $\nu_{0}$, is perturbed under feedback so that the round-trip phase condition is maintained. This emission frequency perturbation influences the laser's optical power, which can be sensed via a photodiode. The laser's terminal voltage also varies in response to feedback [41] providing an alternative signal source that contains equivalent information to the optical power signal $[13,42]$.

Figure 1 shows a diagram of the three-mirror system with the thick lines indicating the beam paths. The beam path external to the laser recombines with the field within the laser in such a way that the phase condition is satisfied in the following equation from [30]: 


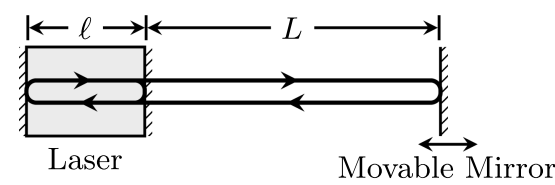

(Target)

Fig. 1. Schematic of the three-mirror configuration. Solid lines and arrows show the beam directions, the reflections from the mirrors, and the transmissions through the partially transparent laser mirrors that are relevant to the model.

$$
0=2 \pi \tau_{\text {ext }}\left(\nu-\nu_{0}\right)+C \sin \left(2 \pi \nu \tau_{\text {ext }}+\arctan \alpha\right),
$$

where $\tau_{\text {ext }}$ is the round-trip time for light in the external laser cavity formed by the remote target, $\nu_{0}$ is the free-running laser emission frequency (without feedback), $\nu$ is the laser emission frequency with feedback, $C$ is the feedback parameter that depends on the amount of light reflected back into the laser $[23,43]$, and $\alpha$ is the linewidth enhancement factor [29] (the ratio between the change in the real and imaginary parts of the refractive index with carrier density [44]). The feedback parameter $C$ can be written as

$$
C=\frac{\tau_{\text {ext }}}{\tau_{\ell}} \kappa_{\text {ext }} \sqrt{1+\alpha^{2}},
$$

where $\tau_{\ell}$ is the round-trip time for light in the laser cavity, and $\kappa_{\text {ext }}$ is the coupling coefficient that depends on the reflectivity of the exit laser facet and the reflectivity of the target, via

$$
\kappa_{\mathrm{ext}}=\varepsilon \sqrt{\frac{R_{\mathrm{ext}}}{R_{s}}}\left(1-R_{s}\right),
$$

with $R_{s}$ and $R_{\text {ext }}$ being the reflectivities of the emitting facet of the laser and of the external target, respectively, and $\varepsilon$ is the fraction of the reflected light coupled back coherently into the lasing mode [23]. Equation (1) makes use of several simplifications. It ignores the effect of the laser gain profile as it is approximately flat within the relatively small perturbations of $\nu$ from $\nu_{0}$. It also ignores the effect of multiple external cavity round-trips (where light reentering the laser is re-emitted and subsequently reenters the laser again). Additionally, it is assumed that the amplitude of light reflected into the laser cavity from the target is much smaller than the amplitude within the laser cavity. This approximation often holds in practice, especially when light is scattered from rough surfaces (e.g., [45] reports an intensity ratio of $10^{-5}$ ). Somewhat counterintuitively, this approximation is justified even when $C$ is greater than one. It is well known that $C$ depends not only on the modal reflection coefficient of the target (the fraction of light coupled back into the lasing mode) but also on the photon lifetime [30] and, consequently, the length of the external cavity. Therefore values of $C$ corresponding to what is termed moderate and strong feedback $(C>1)$ can be readily obtained with only a small fraction of light coupled back into the laser cavity.

The unknown quantity that we wish to obtain from Eq. (1) is the perturbed lasing frequency $\nu$. Equation (1) can be rearranged into a more convenient form by making use of the following substitutions:

$$
\begin{gathered}
\phi=2 \pi \nu \tau_{\text {ext }}, \\
\phi_{0}=2 \pi \nu_{0} \tau_{\text {ext }},
\end{gathered}
$$

where $\phi$ represents the external round-trip phase at the perturbed laser frequency, and $\phi_{0}$ represents the external round-trip phase at the free-running laser frequency. For brevity we will refer to $\phi_{0}$ as simply the external round-trip phase in the remainder of this article.

The substitution of Eqs. ( $\underline{4})$ and (ㅁ) into Eq. ( $\underline{1})$ yields

$$
0=\phi-\phi_{0}+C \sin (\phi+\arctan \alpha) .
$$

In this form, the quantity we wish to solve for is $\phi$. Numerical methods are required to solve Eq. (6) because it has no closed-form solution; a method for solving this equation appears in the next section. There can also be multiple solutions to Eq. (6) when $C$ is greater than one (the precise number of solutions is a function of $C$ and $\left.\phi_{0}[28,37]\right)$. The behavior of the self-mixing system when multiple solutions exist is discussed in Section 3.C.

After solving the phase condition in Eq. (ㅁ) for $\phi$ (described in the next section), the threshold gain change $\Delta g_{\text {th }}$ due to feedback is determined [30]:

$$
\Delta g_{\text {th }}=-\frac{\kappa_{\text {ext }}}{L} \cos \phi
$$

where the coupling coefficient $\kappa_{\text {ext }}$ is defined in Eq. (3), and $L$ is the distance from the laser to the target (see Fig. 1). This equation is used to obtain the change in emitted optical power or laser terminal voltage, either of which can be used as the observable self-mixing signal. The shift in threshold gain will lead to a proportional shift in optical power [40]. Therefore the change in optical power can be written as

$$
\Delta P=\beta \cos (\phi),
$$

where $\beta$ defines the observable self-mixing signal amplitude, which depends on a number of laser and system parameters such as the amount of light being coupled back into the laser cavity, the distance to the target, and the photon lifetime in the laser cavity. In many cases, such as for small displacements, $\beta$ can be treated as a constant. However, $\beta$ can vary significantly over large displacements due to the speckle effect [46] and when measuring the velocity of a rough target. 


\section{Numerical Solution to Phase Condition}

In this section, a procedure for solving the excessphase Eq. (6) is presented, including the case where $C$ is greater than one and there are multiple solutions.

\section{A. Weak Feedback, $C \leq 1$}

When the feedback parameter is less than or equal to one, the right-hand side (r.h.s.) of Eq. (6) is monotonic, and a unique solution can be found for $\phi$. The solution can be found in a robust manner using a bounded root finding algorithm, such as bisection, between known bounds that we denote $\phi_{\min }$ and $\phi_{\max }$. The bounds are obtained by considering the periodicity of the sine function that has a maximum value of +1 and minimum value of -1 . Therefore $\phi_{\min }$ is found by substituting +1 for the sine function in Eq. ( 6 ), and $\phi_{\max }$ by substituting -1 . This yields $\phi_{\min }=\phi_{0}-C$ and $\phi_{\max }=\phi_{0}+C$.

Figure 2 shows a plot of the r.h.s. of Eq. ( $\underline{6})$ and the locations of the bounds, $\phi_{\min }$ and $\phi_{\max }$.

\section{B. Moderate/Strong Feedback, $C>1$}

When the feedback parameter is greater than one, there may be multiple solutions that satisfy Eq. ( $\underline{6})$. Figure $\underline{3}$ shows a plot of the r.h.s. of Eq. ( $\underline{6})$ when this is the case. The boundaries between the increasing and decreasing regions (indicated as solid and broken line segments) are found by setting the derivative of the r.h.s. of Eq. ( $\underline{6})$ to zero and solving for $\phi$ :

$$
\begin{aligned}
0 & =\frac{\partial}{\partial \phi}\left[\phi-\phi_{0}+C \sin (\phi+\arctan \alpha)\right] \\
& =C \cos (\phi+\arctan \alpha)+1
\end{aligned}
$$

Solving Eq. (9) for $\phi$ and using the sign of the second derivative at the solutions to differentiate the peak and trough locations, we obtain

$$
\phi_{\min }=(2 m+1) \pi+\arccos \frac{1}{C}-\arctan \alpha,
$$

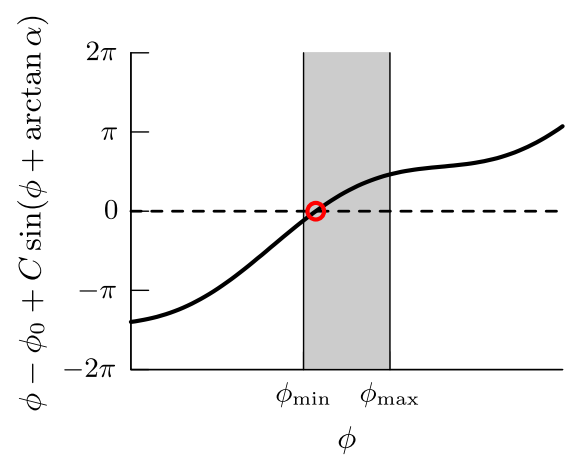

Fig. 2. Plot of the r.h.s. of Eq. (6) for $C=0.8$. The bounds, $\phi_{\min }$ and $\phi_{\max }$, are indicated and supplied to the root finding algorithm to determine the zero crossing (circled).

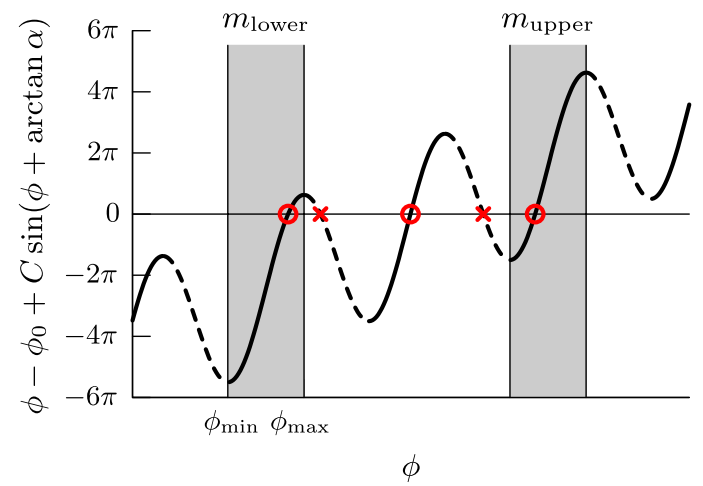

Fig. 3. Plot of the r.h.s. of Eq. (6) for $C=8$. The solid and broken lines indicate the regions where stable and unstable solutions are found, respectively. Circles indicate the stable solutions, and crosses the unstable solutions. Here $m_{\text {lower }}$ and $m_{\text {upper }}$ indicate the regions of the lowest and highest values of $m$ where a solution exists. The bounds, $\phi_{\min }$ and $\phi_{\max }$, have been labeled for the lower region.

$$
\phi_{\max }=(2 m+3) \pi-\arccos \frac{1}{C}-\arctan \alpha,
$$

for each $m$ where $m \in \mathbb{Z}$ (an integer). Figure $\underline{3}$ shows $\phi_{\min }$ and $\phi_{\max }$ corresponding to the trough and peak locations for the left-most solution.

Solutions corresponding to possible lasing modes are indicated by circles and crosses in Fig. 3 , but the modes indicated with crosses are not stable; thus their solutions are not contained here. (Examination of the hysteresis plot in Fig. $\underline{5}$ in Section 3.C gives a strong indication of why this is so, as these unstable modes would never be selected deterministically. The modal stability also can be formally verified though linear stability analysis [28].) Therefore valid solutions are found in the region between the trough and peak for a given $m$.

The next step is to find the possible values of $m$ where a valid solution exists. This is done by considering the lower and upper bounds for $m$, $m_{\text {lower }} \leq m \leq m_{\text {upper. }}$.

\section{Lower Bound}

We obtain $m_{\text {lower }}$ by finding the left-most peak position that is greater than zero. This is obtained by substituting the equation for the peak from Eq. (11) into Eq. ( $\underline{6})$ and rearranging for $m$. As $m$ can only take on integer values, the ceiling (denoted by $\lceil\cdot\rceil$ ) is taken to ensure that the peak value will be greater than zero:

$$
\begin{aligned}
m_{\text {lower }}= & \left\lceil\frac{\phi_{0}+\arctan \alpha+\arccos \frac{1}{C}}{2 \pi}\right. \\
& \left.-\frac{C \sin \left[\arccos \left(\frac{1}{C}\right)\right]}{2 \pi}-\frac{3}{2}\right\rceil .
\end{aligned}
$$

This equation is simplified using a trigonometric identity, $\sin (\arccos a)=\sqrt{1-a^{2}}$, yielding: 


$$
m_{\text {lower }}=\left\lceil\frac{\phi_{0}+\arctan \alpha+\arccos \frac{1}{C}}{2 \pi}-\frac{\sqrt{C^{2}-1}}{2 \pi}-\frac{3}{2}\right\rceil .
$$

\section{Upper Bound}

We obtain $m_{\text {upper }}$ in a similar fashion to the lower bound. It is obtained by finding the right-most trough position that is less than zero. After substituting the equation for the trough from Eq. (10) into Eq. (6) and rearranging for $m$, the floor (denoted by $\lfloor\cdot\rfloor$ ) value is taken to ensure that the trough value will be less than zero:

$$
m_{\text {upper }}=\left\lfloor\frac{\phi_{0}+\arctan \alpha-\arccos \frac{1}{C}}{2 \pi}+\frac{\sqrt{C^{2}-1}}{2 \pi}-\frac{1}{2}\right\rfloor .
$$

\section{Path Dependence (Hysteresis)}

Initially, any valid solution interval could contain $\phi$. In simulation, the choice of initial solution interval is arbitrary without additional information. Once the value of $\phi$ has been determined for a fixed external phase $\phi_{0}$, the solutions as a function of the external phase are considered. This will occur, for example, if a target is moved with respect to the laser, which will be covered in more detail in Section 4.A. Moreover, with a periodic stimulus (such as harmonic motion), $\phi$ will behave periodically from the second period onward.

Figure 4 extends the plot of Fig. 3 to three dimensions with the inclusion of the external phase. This plot provides a visual representation of the r.h.s. of Eq. ( 6 ) and is simply included to assist the reader in forming an intuitive understanding of the solution to Eq. (6). Solutions correspond to the intersection with the plane where $\phi-\phi_{0}+C \sin$ $(\phi+\arctan \alpha)=0$. These solutions are plotted using the thin lines in Fig. 5(b); the thick lines in the figure will be used to illustrate the path dependence of the solutions.

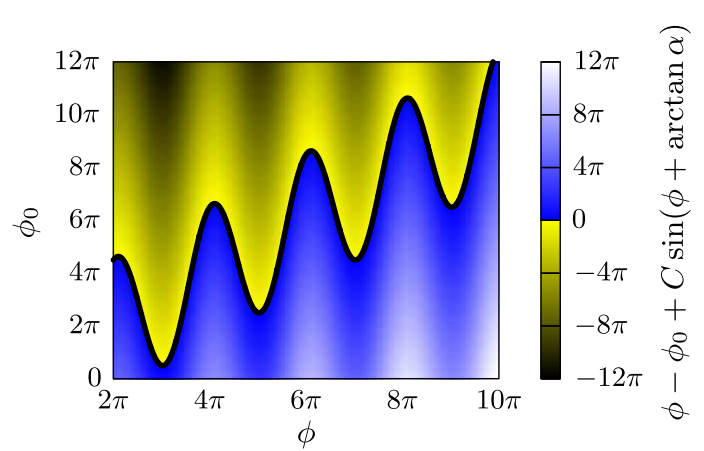

Fig. 4. Surface plot of the r.h.s. of Eq. (6) for a range of values of $\phi$ and $\phi_{0}$ (the external phase) with $\lambda_{0}=\overline{8} 00 \mathrm{~nm}, C=8$, and $\alpha=5$. The locus of points where the function is zero is indicated by the plotted line and corresponds to the solutions of Eq. ( $\underline{6})$.

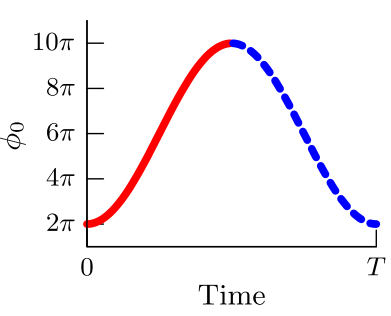

(a)

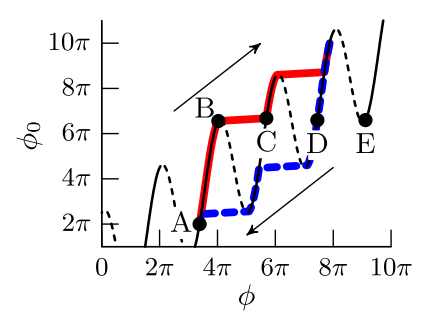

(b)
Fig. 5. Plot (a) shows the sinusoidal external phase function with a period $T$, and plot (b) is the resulting values of $\phi$ for $\lambda_{0}=800 \mathrm{~nm}$, $C=8$, and $\alpha=5$. The thin solid and dotted lines show the possible solutions to the phase equations; the thick solid and thick broken lines trace the locus of solutions in plot (b) to the external phase function in plot (a).

To visualize the hysteresis effect, we consider the solutions for an external phase varying as a harmonic function (as would occur with a target moving in harmonic motion; more details on measuring target displacement are provided in Section 4.A). The external phase is plotted in Fig. 5(a). The path of solutions to the excess-phase equation are then plotted in Fig. 5(b). The thin solid and dotted lines in Fig. $5(\mathrm{~b})$ show the possible solutions for $\phi$ over a range of distances.

As mentioned in the introduction, we restrict ourselves to the stable operating regime in this article. Therefore it follows that, when multiple solutions to Eq. (6) exist, the laser will dwell on one of the solution regions until the solution is no longer valid. This occurs, for example, in the region between A and B in Fig. 5(b) as the target moves away from the laser. As the target continues to move, the solution region no longer contains a valid solution, so the lasing mode could potentially jump to solutions at points $\mathrm{C}, \mathrm{D}$, or $\mathrm{E}$ in Fig. 5(b). However, we assume the laser will jump to the next closest solution (in this case, point $\mathrm{C}$ ); this is the behavior we have most often observed and the behavior that is reported in most of the self-mixing literature (although a different behavior has also been observed for a specific model of laser where a solution is skipped and jumps to the subsequent solution [47]). The parameters that determine this behavior are not yet known, and it has been suggested that this phenomenon is related to semiconductor laser dynamics [47].

Relating these assumptions on the laser behavior to the equations derived above, if a solution previously existed for a given integer value of $m$, the solution will remain in the same region until $m$ falls outside of $\left[m_{\text {lower }}, m_{\text {upper }}\right]$. When a solution does disappear, the laser frequency will usually hop to the next closest region with a valid solution; either $m_{\text {lower }}$ (when $m<m_{\text {lower }}$ ) or $m_{\text {upper }}$ (when $m>m_{\text {upper }}$ ). This is used in the logic developed in the pseudocode for solving the excess-phase equation in Algorithm 1.

\section{Solving for Power}

After solving the phase condition to obtain $\phi$, the change in power is obtained directly from Eq. (8). 


\section{E. Pseudocode for Solving Self-Mixing Equations}

The pseudocode in Algorithm 1, based on the previous discussion, provides a systematic method to find the power variations expected for a given feedback parameter $C$, free-running laser external phase $\phi_{0}$, and laser linewidth enhancement factor $\alpha$. A suitable zero finding function is required that must search for a solution within the given bounds (FINDZERO in the pseudocode). MATLAB's fzero root finding function has been used successfully and converges rapidly by using a combination of bisection, secant, and inverse quadratic interpolation methods [48]. We have included a MATLAB code listing that implements the pseudocode in Algorithm 1 labeled selmixpower.m (Listing 1 in Appendix A).

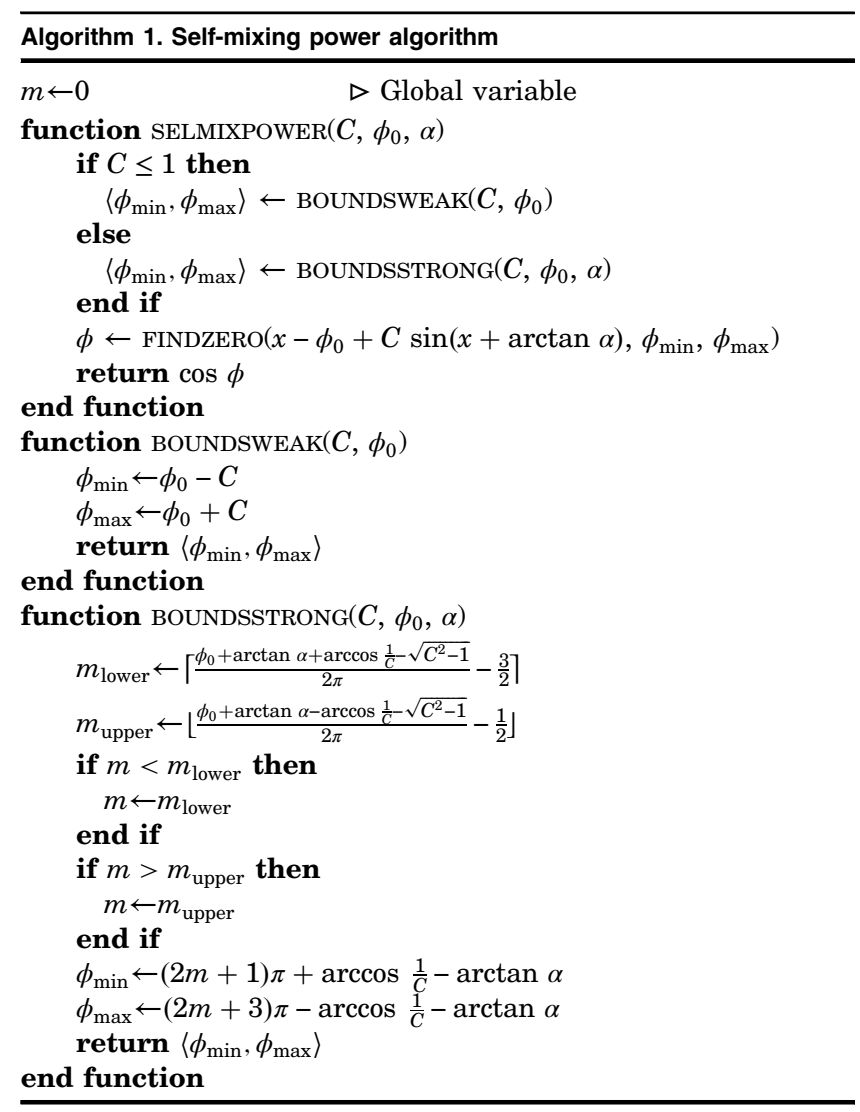

One subtlety must be considered when using MATLAB's fzero function. The values at the bounds must differ in sign. In rare cases, one of the bounds may lie close to the solution (within the termination criteria tolerances), but, due to numerical error, the value at this point does not have the correct sign. This issue can be taken care of by testing the values at the bounds and using the lower bound as the result if its value's sign is positive or using the upper bound as the result if its value's sign is negative; otherwise, the fzero function is called normally.

Algorithm 2 presents pseudocode to synthesize self-mixing signal waveforms for given round-trip phase samples in vector $\phi_{0}$, which demonstrates how to use the selmixpower function from Algorithm 1.
Here $N$ is the number of samples to be obtained, $T$ is the total simulation time, $\mathbf{t}$ is a vector containing the sample times (evenly spaced here), and $\mathbf{p}$ is a vector containing the samples of the laser power over time (i.e., the synthetic self-mixing signal). This algorithm will form the basis used to demonstrate how to generate self-mixing signals for a range of selfmixing sensor configurations.

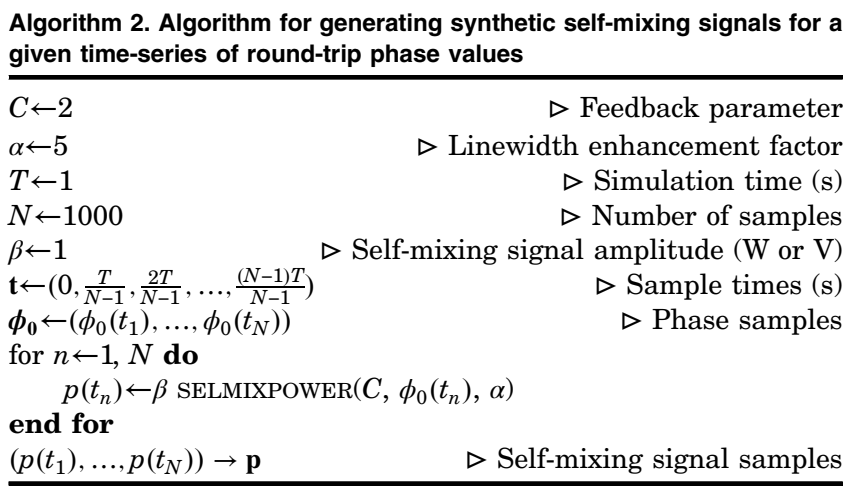

\section{Applications}

This section demonstrates how to apply Algorithms 1 and 2 developed in the previous section to typical self-mixing sensor configurations. The examples presented aim to provide a starting point for self-mixing laser modeling that can be extended to other selfmixing sensor applications with little effort.

\section{A. Target Displacement}

Measuring target displacement is an application often presented in the published self-mixing literature, as it is easy to understand and makes use of simple stimuli. This section will describe how to generate synthetic self-mixing target displacement signals.

In addition to their generation, these synthetic signals can be fitted to experimental target displacement signals. In practice, this is usually achieved by varying the self-mixing model parameters and computing a measure of distance or discrepancy between the modeled and experimental signals. This process permits the parameters associated with experimental signals to be estimated, such as the feedback parameter $C$ [36]. Moreover, the self-mixing algorithm described here has been used recently to aid in parameter extraction for self-mixing signals acquired from a terahertz quantum cascade laser [49].

Target displacement causes a proportional change in the external phase $\phi$. For illustration, consider a target that is displaced a distance $d$ from a nominal position $L_{0}$. From Eq. (5), the sampled external round-trip phase for the free-running laser as a function of the target distance is given by

$$
\phi_{0}\left(t_{n}\right)=\frac{4 \pi \nu_{0}}{c}\left[L_{0}+d\left(t_{n}\right)\right],
$$

where $c$ denotes the speed of light in vacuum. 
The displacement samples can be an arbitrary function of time. However, we provide a concrete example with harmonic motion displacement samples with an amplitude $A$ and frequency $f$. Therefore

$$
d\left(t_{n}\right)=A \cos \left(2 \pi f t_{n}\right)
$$

Substituting Eq. (16) into Eq. (15) and using the relationship $c=\nu_{0} \lambda_{0}$ (where $\lambda_{0}$ is the free-running laser wavelength) yields

$$
\phi_{0}\left(t_{n}\right)=\frac{4 \pi}{\lambda_{0}}\left[L_{0}+A \cos \left(2 \pi f t_{n}\right)\right],
$$

which can then be used with Algorithm 2 to generate synthetic self-mixing signals. MATLAB code that generates synthetic self-mixing signals for a target moving in harmonic motion is given in harmonic motion.m (Listing 2 in Appendix $\underline{A}$ ). The results from this code are plotted in Fig. 6 for a range of values of the feedback parameter. We can observe trends in the evolution of the self-mixing signal for increasing values of the feedback parameter $C$; the hysteresis effect becomes greater, waveform asymmetry becomes more pronounced, and the number of fringes

(a)

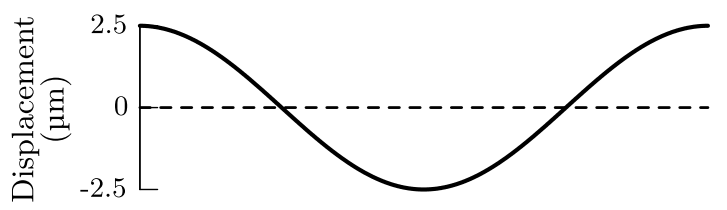

(b)

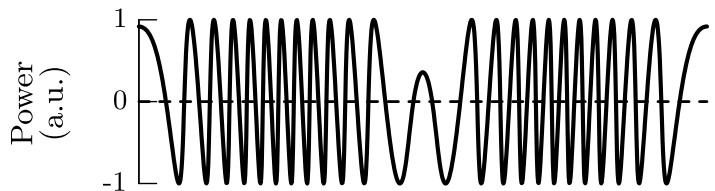

(c)

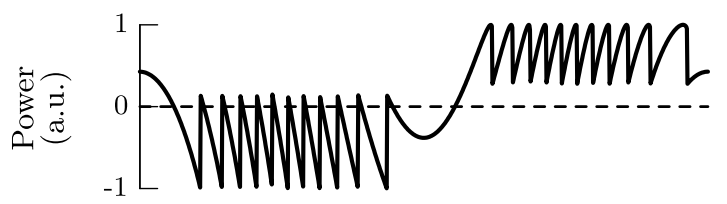

(d)

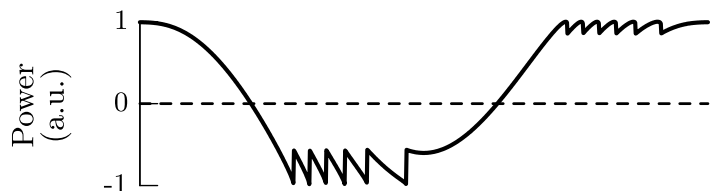

(e)

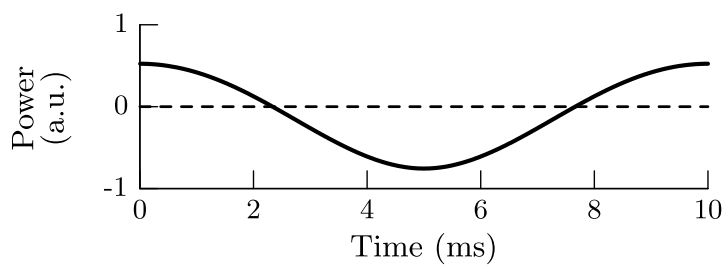

Fig. 6. Plot (a) shows the target displacement motion used to generate the synthetic self-mixing signal in plots (b)-(e) using the provided MATLAB code with a laser wavelength of $850 \mathrm{~nm}$ and a target moving in harmonic motion with an amplitude of $2.5 \mu \mathrm{m}$ at a frequency of $100 \mathrm{~Hz}$. Plots (b)-(e) show the evolution of the self-mixing signals as the feedback parameter, $C$, increases through $0.5,5,20$, and 60 . is decreased. It is interesting to note that fringes disappear completely for sufficiently large values of the feedback parameter, a situation in which the stimulus is replicated in the self-mixing waveform.

An example of the model applied to experimental data is shown in Fig. 7. The experimental data was acquired from the junction voltage variations of a VCSEL with an $850 \mathrm{~nm}$ wavelength. The plots show good agreement between the experimental and synthetic self-mixing signals.

\section{B. Absolute Distance}

The distance to a fixed target can be obtained from the self-mixing sensor by frequency modulating the laser. The frequency modulation is typically achieved by modulating the laser bias current with an ostensibly triangular waveform [6]. In practice, the laser frequency does not change linearly with current, and the triangle waveform is usually predistorted to compensate for thermal and other effects to produce a linear frequency sweep [38,50]. However, for illustrative purposes we will assume a linear link between current changes and frequency changes.

The self-mixing signal from the absolute distance sensor is modeled by first calculating the frequency modulation waveform

$$
\Delta \nu\left(t_{n}\right)=\Delta F \operatorname{Tri}\left(t_{n}\right)
$$

where Tri represents a triangle function, and $\Delta F$ is the frequency modulation coefficient. If we denote

(a)

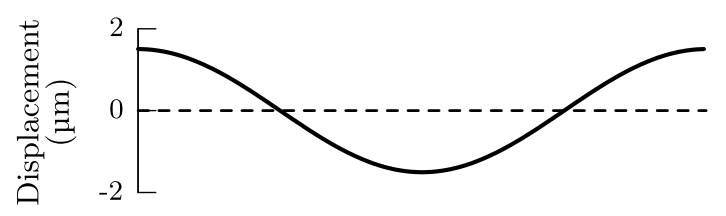

(b)

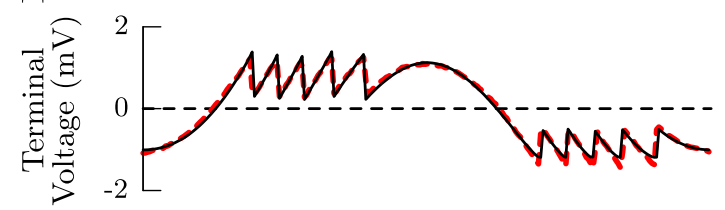

(c)

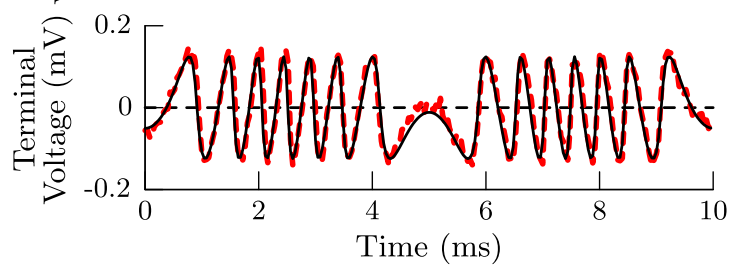

Fig. 7. Plots showing the agreement between experimental selfmixing displacement signals and the corresponding fits to synthetic self-mixing signals generated using the algorithm described in this article. The target displacement is plotted in (a) and is relative to a nominal position of $100 \mathrm{~mm}$ from the laser. Plots (b) and (c) show the experimental signals (broken lines) and the corresponding synthetic self-mixing signals (solid lines). A moderate feedback signal was obtained in plot (b) $(C=6.8)$ while a weak feedback signal was obtained in plot (c) $(C=0.51)$ by introducing a neutral density filter into the beam path. The signal was acquired from the terminal voltage variations of an $850 \mathrm{~nm}$ wavelength VCSEL (Litrax LX-VCS-850-T101). 
the unmodulated laser frequency without feedback as $\nu_{0}^{\prime}$, then the modulated laser frequency without feedback is now given by

$$
\nu_{0}\left(t_{n}\right)=\nu_{0}^{\prime}+\Delta \nu\left(t_{n}\right)=\nu_{0}^{\prime}+\Delta F \operatorname{Tri}\left(t_{n}\right) .
$$

The external phase can thus be obtained using Eq. (ㅁ) giving

$$
\phi_{0}\left(t_{n}\right)=\frac{4 \pi\left[\nu_{0}^{\prime}+\Delta F \operatorname{Tri}\left(t_{n}\right)\right] L}{c} .
$$

Making use of the relationship $c=\nu_{0}^{\prime} \lambda_{0}$, we can rewrite this as

$$
\phi_{0}\left(t_{n}\right)=4 \pi L\left[\frac{1}{\lambda_{0}}+\frac{\Delta F \operatorname{Tri}\left(t_{n}\right)}{c}\right] .
$$

A side effect of the laser current modulation is modulation of the laser output power. We model this by adding a triangle waveform to the resulting selfmixing power variations with an amplitude equal to a power modulation coefficient $\rho$ :

$$
p\left(t_{n}\right)^{\prime}=p\left(t_{n}\right)+\rho \operatorname{Tri}\left(t_{n}\right) .
$$

Equations (21) and (22) can be used in Algorithm 2 to generate the synthetic absolute distance signals. MATLAB code implementing the algorithm appears in absolute_distance.m (Listing 3 in Appendix A) with the resulting signal plotted in Fig. 8 for a target distance of $24 \mathrm{~mm}$ and a laser frequency sweep over a range of $46 \mathrm{GHz}$ with a base frequency of $\nu_{0}^{\prime}=c /(845 \mathrm{~nm})$. In addition to the synthetic signal, Fig. 8 also contains an experimental signal from [6] showing good agreement. We can perform a rough (a)

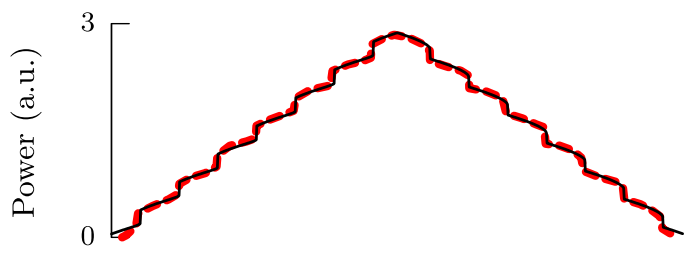

(b)

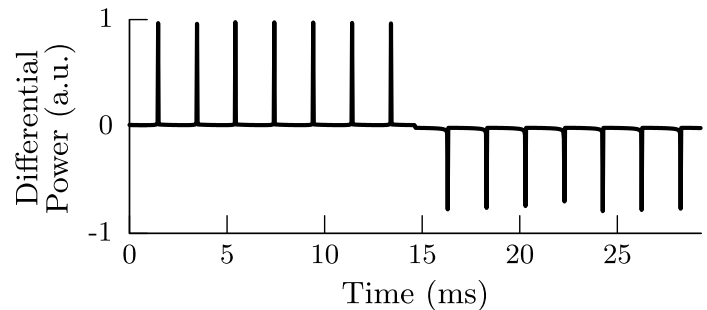

Fig. 8. Plot (a) shows synthetic (solid line) and experimental (broken line) absolute distance self-mixing sensor signals. Plot (b) shows the result of numerically differentiating the synthetic signal in (a). The experimental signal was extracted from [6, Fig. 2(a)]. The synthetic signal target distance was $24 \mathrm{~mm}$ plus a small offset of $0.5 \mu \mathrm{m}$ to align the synthetic signal fringe positions with the experimental fringe positions. The laser frequency sweep range is $46 \mathrm{GHz}$. The modulation period of the triangle waveform is $29.3 \mu \mathrm{s}$. check on the synthetic signal using the result derived by Beheim and Fritsch [6]: $L=N_{\mathrm{f}} c /(2 \Delta F)$ with an uncertainty of $c /(2 \Delta F)$ where $N_{\mathrm{f}}$ is the number of fringes observed in the signal. With seven fringes in Fig. $8, L=22.8 \pm 3.3 \mathrm{~mm}$, which includes the modeled value of $L=24 \mathrm{~mm}$.

Apart from the determination of absolute distance, linear frequency modulation of the laser also has been used to interrogate the complex refractive index of a stationary remote target [51].

\section{Absolute Distance and Velocity}

It is also possible to consider the previous absolute distance measurement with a target in motion. This scenario provides a link to the next section that considers the case of the self-mixing velocimetry sensor. In contrast to the next section that considers a rough target that scatters light in all directions, in this section we continue to consider a target that will reflect light directly back into the laser.

For the purposes of simulation, we consider the target at position $L_{0}$ when $t=0$, traveling with a velocity $v$, away from the laser; therefore

$$
L\left(t_{n}\right)=L_{0}+v t_{n} .
$$

Apart from this additional consideration, the processes for generating the synthetic self-mixing signal are the same as in the previous section. Substituting Eq. (23) into Eq. (21), we obtain the expression for the external phase,

$$
\phi_{0}\left(t_{n}\right)=4 \pi\left(L_{0}+v t_{n}\right)\left[\frac{1}{\lambda_{0}}+\frac{\Delta F \operatorname{Tri}\left(t_{n}\right)}{c}\right] .
$$

The inclusion of the velocity term accounts for the Doppler effect caused by the target motion. Equations (22) and (24) can be used in Algorithm 2 to generate the synthetic self-mixing signals. MATLAB code implementing the algorithm appears in absolute_distance+velocity.m (Listing 4 in Appendix $\underline{\text { A) }}$ with the resulting signal plotted in Fig. $\underline{9}$ with a target velocity of $0.3 \mathrm{~mm} / \mathrm{s}$ (the other parameters are the same as in the previous section). The target velocity leads to an asymmetry in the number of fringes observed in each half of the triangle waveform. Moreover, due to the speed at which the external cavity is continuously extended, all of the spikes observed in Fig. 9(b) are negative (unlike in the previous section).

A self-mixing distance and velocity sensor has been discussed by de Groot and Gallatin, who show that the resulting spectrum of the self-mixing signal contains information about the target velocity and distance [52]. The spectrum consists of two peaks whose frequency separation is related to the target distance, and their average frequency is related to the target velocity.

Velocity sensing using the self-mixing effect is explored further in the next section where the effect of surface roughness is also considered. 
(a)

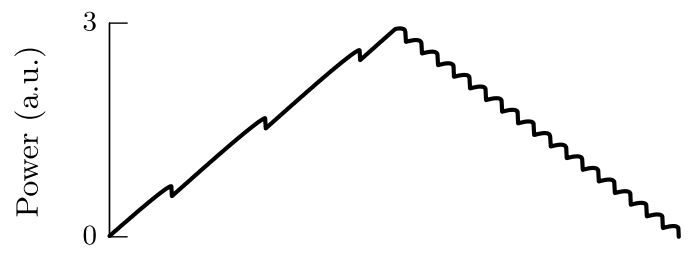

(b)

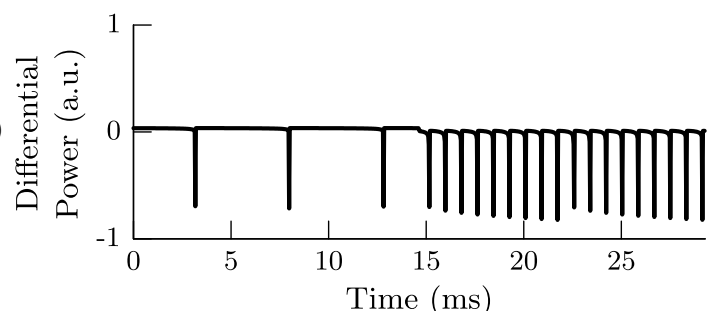

Fig. 9. Plot (a) shows the synthetic absolute distance and velocity self-mixing sensor signal, which is numerically differentiated in plot (b) for a target with a velocity of $0.3 \mathrm{~mm} / \mathrm{s}$ at a distance of $24 \mathrm{~mm}$ and a laser frequency sweep over a range of $46 \mathrm{GHz}$. The modulation period of the triangle waveform is $29.3 \mu \mathrm{s}$.

\section{Velocimetry with Rough Target}

In previous applications in this article, we dealt with simple deterministic external phase stimuli. This was sufficient to well replicate the experimental results. The self-mixing sensor can also provide useful signals for nondeterministic stimuli, which is of interest, for example, for sensing the velocity of a rough moving target $[12,45]$.

In this final example, we demonstrate how a nondeterministic time-domain stimulus can be coupled with the excess phase equation. The nondeterministic signal that we synthesize is consistent with those observed in typical self-mixing velocimetry-sensing experiments where the moving target is rough compared with the laser wavelength. The interaction of the light with the rough surface produces a complex valued random process in time with amplitude and phase fluctuations [53]. This nondeterministic stimulus then serves as the input to the excess phase solver, as we have done in previous sections. To this end, we represent the phenomenological elements leading to Doppler shift and the broadening of the signal in the frequency domain. We propose a mathematical procedure to create a time-domain signal consistent with both the experimental time-domain signal and its spectrum. This ensures the mutual consistency between the time- and frequency-domain realizations associated with this nondeterministic stimulus.

Therefore, without going into the complexities of underlying physical phenomena, we use a Gaussian power spectral density (PSD) to describe the random process that will be input to the excess phase solver:

$$
S(f)=\exp \left[-\frac{4 \log _{e} 2\left(f-f_{D}\right)^{2}}{\mathrm{FWHM}^{2}}\right],
$$

where $S(f)$ is the resulting $\mathrm{PSD}, f_{D}$ is the center or Doppler frequency, and FWHM is the full width at half-maximum of the Doppler peak. This PSD determines the width of the fundamental peak of the Doppler spectrum. Noting that the corresponding phase is uniformly distributed, this PSD can be straightforwardly extended to the complex plane. The inverse Fourier transform of this complex function can be efficiently generated using fast Fourier transform (FFT) techniques [54-56]. We take the resulting complex (time-domain) samples, denoted by $\psi\left(t_{n}\right)$, as our stimulus.

In the previous applications, the level of feedback was fixed; hence the feedback parameter, $C$, was also fixed. In contrast, this nondeterministic stimulus leads to a modulation of $C$. As $\beta$ is proportional to $\kappa_{\text {ext }}$ defined in Eq. (3), which is in turn proportional to $C$, this simultaneously leads to a modulation of $\beta$. This can be incorporated into the self-mixing model by modulating the feedback parameter and $\beta$ by the amplitude of the stimulus $\psi\left(t_{n}\right)$. Based on these considerations, we can update the line in Algorithm 2 from

$$
p\left(t_{n}\right) \leftarrow \beta \text { SELMIXPOWER }\left(C, \phi_{0}\left(t_{n}\right), \alpha\right)
$$

to

$$
p\left(t_{n}\right) \leftarrow \beta\left|\psi\left(t_{n}\right)\right| \operatorname{SELMIXPOWER}\left(C_{0}\left|\psi\left(t_{n}\right)\right|, \phi_{0}\left(t_{n}\right), \alpha\right),
$$

where $C_{0}$ represents the nominal value of $C$. The phase samples of the re-injected light, $\phi_{0}\left(t_{n}\right)$, are generated from the phase of the realization of the random process as follows:

$$
\phi_{0}\left(t_{n}\right)=\frac{4 \pi L_{0}}{\lambda}+\arg \left[\psi\left(t_{n}\right)\right]
$$

where the phase term corresponding to the roundtrip distance to the target at distance $L_{0}$ has been retained from the target displacement application and may be additionally modulated to capture the effect of concurrently changing the distance between the laser and the target.

The procedure for simulating the self-mixing velocimetry sensor is codified as follows:

1. A realization of the stimulus signal is created based on the PSD of the random process given by Eq. (25). This is achieved by imposing random complex phase fluctuations on the PSD and taking the inverse FFT operation (denoted IFFT) as follows:

$$
\psi\left(t_{n}\right)=\operatorname{IFFT}\left[S\left(f_{n}\right) \exp \left(i \vartheta\left(f_{n}\right)\right)\right]
$$

where $i=\sqrt{-1}$, and $\vartheta\left(f_{n}\right)$ is the $n$th independent realization of a uniformly distributed random variable over $[-\pi, \pi]$. The stimulus signal time samples, $\psi\left(t_{n}\right)$, are in the complex domain and represent the amplitude and phase fluctuations of the stimulus.

2 . The round-trip phase samples, $\phi_{0}\left(t_{n}\right)$, are generated from the phase of $\psi\left(t_{n}\right)$ according to Eq. (26). 
3. The self-mixing power variation samples, $p\left(t_{n}\right)$, are calculated according to Algorithm 2 with the modifications mentioned.

4. The laser noise is modeled by adding (uniform frequency) white noise to the power variation samples.

5 . The PSD of the synthetic noisy self-mixing power variations is estimated (in the MATLAB code listing we use Welch's method [57]).

A MATLAB script has been provided that implements these steps in velocimetry.m (Listing 5 in Appendix A). Figure 10 plots synthetic time-domain signals generated by this script. Figure 11 shows the estimated PSD generated by the script along with an experimentally obtained self-mixing velocimetry signal. (The experimental signal was obtained using the experimental setup described in [53]. An $850 \mathrm{~nm}$ VCSEL was employed with a sandblasted aluminum spinning target with a root-mean-square height variation of several times the optical wavelength.) The parameter values of the script were chosen to provide a good fit to the experimental signal, and the resulting PSDs were normalized.

The reader should consider the algorithm described above as a convenient tool for synthesizing self-mixing Doppler spectra, which closely resemble the experiment. A more complex model will surely be required to explain the underlying physical effects that are responsible for this outcome. Nevertheless, this procedure provides means for extracting parameter values, such as the feedback parameter, $C$, by fitting experimentally acquired signals to the synthetic velocimetry signal. For the present example, $C=0.36$, a value consistent with both the time

(a)

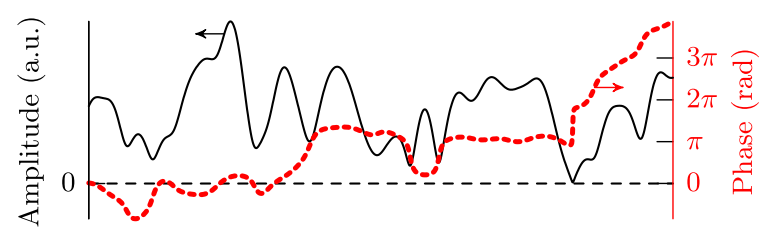

(b)

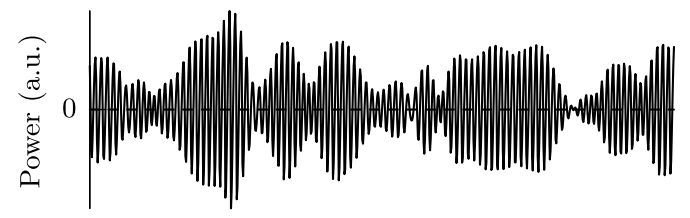

(c)

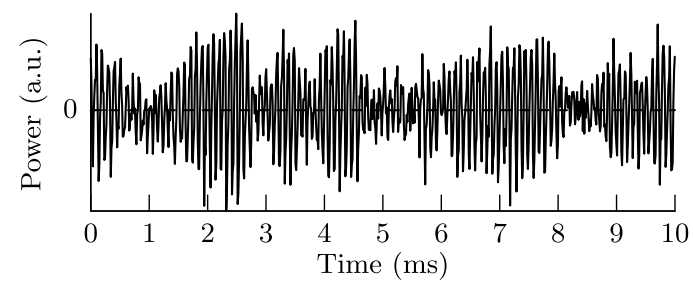

Fig. 10. Plots from various stages in the generation of a synthetic self-mixing velocimetry signal. The plots in (a) show the amplitude (solid line) and unwrapped phase (broken line) of a realization of a random process that models the beam scattered from a rough target. (The linear component of the unwrapped phase has been removed to make the phase variations visible.) Plot (b) is the self-mixing signal obtained prior to adding noise. Plot (c) is the signal after white noise has been added to model the laser intensity noise.

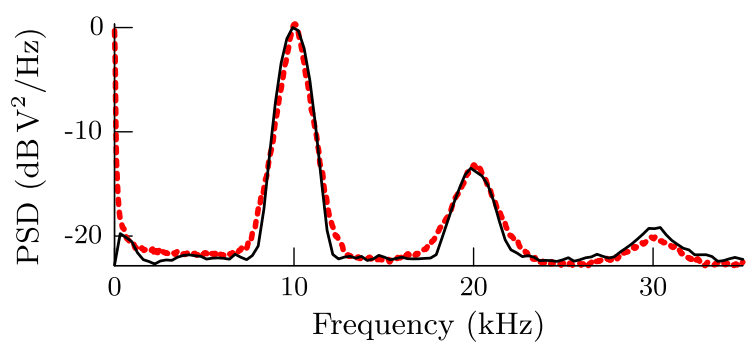

Fig. 11. PSD of the self-mixing power variations obtained from the velocimetry simulation (solid line) along with an experimentally obtained signal for comparison (broken line).

domain signals that show little asymmetry and the $13.5 \mathrm{~dB}$ suppression of the second harmonic of the spectrum in Fig. 11.

\section{E. Other Applications}

The procedure for solving the excess-phase equation presented in this article also can be applied to a range of other applications where various parameters change over time. One example is the selfmixing imaging sensor where an optical chopper is used to modulate the self-mixing signal [13]. The chopper can be modeled by considering two states: the chopper obstructing the beam, and the chopper allowing the beam to pass through. These states would correspond to changing the feedback parameter (when the chopper obstructs the beam, the feedback would be reduced) and the optical path length (which would be equal to the distance between the laser and the chopper when the beam is obstructed by the chopper). Again, Algorithm 2 can be used to generate the synthetic signal for this case if the time series of the round-trip phase and feedback parameters are provided.

\section{Conclusions}

This article presents a simple, systematic method for solving the excess-phase equation numerically to generate synthetic self-mixing signals for a range of feedback levels. This model of the self-mixing sensor can be applied regardless of the feedback regime and has been demonstrated to be general enough to apply to a wide range of self-mixing sensor applications, including target displacement measurement, absolute distance measurement, and velocimetry. MATLAB code listings that generate synthetic signals for the different sensor applications are included in Appendix A.

The ability to synthesize self-mixing sensor signals can provide insight into the operation and performance of self-mixing sensors under different experimental conditions. Moreover, such synthetic signals can be fitted to experimentally observed signals, enabling the extraction of independent experimental system parameters. This simple, systematic method for solving the excess-phase equation and generating synthetic self-mixing signals can be coupled with additional physical models of other parts of a sensor system, enabling a more complete and accurate model of sensor behavior. 
$\%$ Functions for solving self-mixing equations-Kliese et al., 2014

function power $=$ selmixpower $(\mathrm{C}$, phi0, alpha $) \%$ Power level at a sample in time

if $(\mathrm{C}<=1.0)$

else

[phimin, phimax] = boundsweak $(\mathrm{C}$, phi0);

end

[phimin, phimax] = boundsstrong $(\mathrm{C}$, phi0, alpha $)$;

excessphase $=@(x) x-\operatorname{phi} 0+C * \sin (x+\operatorname{atan}($ alpha $))$;

$\%$ If the value at the left bound positive, then it will be very close to the solution.

$\%$ If the value at the upper bound is negative, it will be very close to the solution.

if (excessphase (phimin) $>0$ )

excessphase (phimin)

phi = phimin;

elseif (excessphase (phimax) $<0)$

excessphase (phimax)

phi $=$ phimax;

else

end

phi $=$ fzero $($ excessphase, $[$ phimin, phimax $]) ;$

power $=\cos ($ phi)

end

function [phimin, phimax] $=$ boundsweak $(\mathrm{C}$, phi0) $\%$ Find search region when $\mathrm{C}<=1$

phimin = phi0 $-\mathrm{C}$;

phimax $=$ phi0 $+\mathrm{C}$;

end

function [phimin, phimax] $=$ boundsstrong $(\mathrm{C}$, phi0, alpha) $\%$ Find search region when $\mathrm{C}>=1$

persistent $\mathrm{m}$; \% Solution region number

if isempty $(\mathrm{m}) ; \mathrm{m}=0$; end

$\%$ Calculate upper \& lower values of $\mathrm{m}$ where solutions exist then ensure $\mathrm{m}$ is between them

mlower $=$ ceil $\left(\left(\right.\right.$ phi0 $+\operatorname{atan}($ alpha $\left.)+\operatorname{acos}(1 / \mathrm{C})-\operatorname{sqrt}\left(\mathrm{C}^{*} \mathrm{C}-1\right)\right) /\left(2^{*}\right.$ pi $\left.)-1.5\right)$;

mupper $=$ floor $\left(\left(\right.\right.$ phi $0+$ atan $($ alpha $\left.\left.)-\operatorname{acos}(1 / \mathrm{C})+\operatorname{sqrt}\left(\mathrm{C}^{*} \mathrm{C}-1\right)\right) /\left(2^{*} \mathrm{pi}\right)-0.5\right)$;

if $(\mathrm{m}<$ mlower $) ; \mathrm{m}=$ mlower; end

if ( $\mathrm{m}>$ mupper); $\mathrm{m}=$ mupper; end

phimin $=(2 * \mathrm{~m}+1) * \mathrm{pi}+\operatorname{acos}(1 / \mathrm{C})-\operatorname{atan}($ alpha $) ; \%$ Trough

phimax $=(2 * \mathrm{~m}+3) *$ pi $-\operatorname{acos}(1 / \mathrm{C})-\operatorname{atan}($ alpha $) ; \%$ Peak

end

Listing 2. harmonic_motion.m

$\%$ Example self-mixing signal generation for harmonic motion-Kliese et al., 2014

$\mathrm{C}=2$;

alpha $=4.6$;

$\mathrm{T}=20 \mathrm{e}-3$

$\mathrm{N}=2000$

beta $=1$;

lambda $0=850 \mathrm{e}-9$

$\mathrm{L} 0=0.1$

$\mathrm{A}=2.5 \mathrm{e}-6$;

$\mathrm{f}=100$;

$\mathrm{t}=0: \mathrm{T} /(\mathrm{N}-1): \mathrm{T}$;

$\mathrm{d}=\mathrm{A} * \cos \left(2^{*} \mathrm{pi}^{*} \mathrm{f}^{*} \mathrm{t}\right)$

phi0 $=4^{*}$ pi/lambda $0 *(\mathrm{~L} 0+\mathrm{d})$;

$\mathrm{p}=\operatorname{zeros}(1, \mathrm{~N})$;

for $\mathrm{i}=1: \mathrm{N}$$$
\mathrm{p}(\mathrm{i})=\text { beta } * \operatorname{selmixpower}(\mathrm{C}, \text { phi0 }(\mathrm{i}), \text { alpha }) \text {; }
$$

end

plot (t, p); xlabel ('Time (s)'); ylabel ('Power Variation ');
$\%$ Feedback parameter

$\%$ Linewidth enhancement factor

$\%$ Simulation time (s)

$\%$ Number of samples

$\%$ Laser power modulation coefficient

$\%$ Laser wavelength $(\mathrm{m})$

$\%$ Target nominal distance (m)

$\%$ Motion amplitude (m)

$\%$ Motion frequency $(\mathrm{Hz})$

$\%$ Sample times

$\%$ Displacement samples

$\%$ Round-trip phase samples

$\%$ Self-mixing signal samples

$\%$ Generate the synthetic self-mixing signal

$\%$ Plot the results

Listing 3. absolute_distance.m

$\%$ Example self-mixing signal generation for a modulated laser-Kliese et al., 2014

$\mathrm{c}=299792458$;

$\mathrm{C}=2$;

alpha $=4.6$;

$\mathrm{T}=29.3 \mathrm{e}-3$;
$\%$ Speed of light in vacuum $(\mathrm{m} / \mathrm{s})$

$\%$ Feedback parameter

$\%$ Linewidth enhancement factor

$\%$ Simulation time (s)

(Listing continued) 
$\mathrm{N}=1000$

beta $=0.1$

lambda $0=845 \mathrm{e}-9$;

$\mathrm{L}=0.024+0.5 \mathrm{e}-6$

deltaf $=-46 \mathrm{e} 9$;

$\mathrm{pm}=2.9$;

$\mathrm{t}=0: \mathrm{T} /(\mathrm{N}-1): \mathrm{T}$;

triper $=\mathrm{T}$;

tri $=1+\operatorname{sign}(\bmod (\mathrm{t} /$ triper, 1$)-0.5) *\left(1-2^{*} \bmod (\mathrm{t} /\right.$ triper, 1$\left.)\right)$;

phi $0=4 *$ pi $* L^{*}(1 /$ lambda $0+$ deltaf $*$ tri $/ \mathrm{c})$;

$\mathrm{p}=\operatorname{zeros}(1, \mathrm{~N})$;

for $\mathrm{i}=1: \mathrm{N}$ end

$\mathrm{p}(\mathrm{i})=$ beta $*$ selmixpower $(\mathrm{C}$, phi0 $(\mathrm{i})$, alpha $)+\mathrm{pm} * \operatorname{tri}(\mathrm{i}) ;$

plot (t, p); xlabel ('Time (s)'); ylabel ('Power Variation ');
$\%$ Number of samples

$\%$ Laser power modulation coefficient

$\%$ Laser wavelength $(\mathrm{m})$

$\%$ Target distance $(\mathrm{m})$

$\%$ Frequency modulation coefficient $(\mathrm{Hz})$

$\%$ Power modulation coefficient

$\%$ Sample times

$\%$ Triangle period (s)

$\%$ Triangle waveform

$\%$ Round-trip phase samples

$\%$ Self-mixing signal samples

$\%$ Generate the synthetic self-mixing signal

$\%$ Plot the results

\section{Listing 4. absolute_distance+velocity.m}

$\%$ Example self-mixing signal generation for a modulated laser with the target

$\%$ moving with a constant velocity—Kliese et al., 2014

$\mathrm{c}=299792458$

$\mathrm{C}=2$

alpha $=4.6$;

$\mathrm{T}=29.3 \mathrm{e}-3$

$\mathrm{N}=1000$;

beta $=0.1$

lambda $0=845 \mathrm{e}-9$

$\mathrm{L} 0=0.024$

$\mathrm{v}=300 \mathrm{e}-6$

deltaf $=-46 \mathrm{e} 9$

$\mathrm{pm}=2.9$;

$\mathrm{t}=0: \mathrm{T} /(\mathrm{N}-1): \mathrm{T}$

triper $=\mathrm{T}$;

tri $=1+\operatorname{sign}(\bmod (\mathrm{t} /$ triper, 1$)-0.5) *\left(1-2^{*} \bmod (\mathrm{t} /\right.$ triper, 1$\left.)\right)$;

phi $0=4^{*}$ pi $*(\mathrm{~L} 0+\mathrm{v} * \mathrm{t}) *(1 /$ lambda $0+$ deltaf $* \operatorname{tri} / \mathrm{c})$;

$\mathrm{p}=\operatorname{zeros}(1, \mathrm{~N})$;

for $\mathrm{i}=1: \mathrm{N}$

$\mathrm{p}(\mathrm{i})=$ beta $*$ selmixpower $(\mathrm{C}$, phi0 $(\mathrm{i})$, alpha $)+$ pm*tri(i); end

plot (t, p); xlabel ('Time (s)'); ylabel ('Power Variation ');

$\%$ Speed of light in vacuum $(\mathrm{m} / \mathrm{s})$

$\%$ Feedback parameter

$\%$ Linewidth enhancement factor

$\%$ Simulation time (s)

$\%$ Number of samples

$\%$ Laser power modulation coefficient

$\%$ Laser wavelength (m)

$\%$ Target start distance $(\mathrm{m})$

$\%$ Target velocity $(\mathrm{m} / \mathrm{s})$

$\%$ Frequency modulation coefficient $(\mathrm{Hz})$

$\%$ Power modulation coefficient

$\%$ Sample times

$\%$ Triangle period (s)

$\%$ Triangle waveform

$\%$ Round-trip phase samples

$\%$ Self-mixing signal samples

$\%$ Generate the synthetic self-mixing signal

$\%$ Plot the results

Listing 5. velocimetry.m

$\%$ Example self-mixing velocimetry signal generation-Kliese et al., 2014

$\mathrm{C} 0=0.36$;

alpha $=4.6$

$\mathrm{T}=0.8$

$\mathrm{N}=2 \wedge 16$;

beta $=1$

lambda $0=850 \mathrm{e}-9$

$\mathrm{L} 0=0.1$

fwhm $=1800$

$\mathrm{fD}=10000$

noise $=0.60$

$\mathrm{t}=0: \mathrm{T} /(\mathrm{N}-1): \mathrm{T}$

$\mathrm{p}=\operatorname{zeros}(1, \mathrm{~N})$;

$\%$ Generate a realization of the random process, psi, from the power spectral

$\%$ density, S, corresponding to the random speckle process (scaled to have

$\%$ an RMS value of 1 after generating the realization of the random process).

$\mathrm{f}=0: 1 / \mathrm{T}:(\mathrm{N}-1) / \mathrm{T}$;

$\mathrm{S}=\mathrm{N}^{*} \operatorname{sqrt}\left(2^{*} \operatorname{sqrt}\left(2^{*} \log (2)\right) /\left(\operatorname{sqrt}(\mathrm{pi})^{*} \mathrm{~T}^{*} \mathrm{fwhm}\right)\right)^{*} \exp \left(-4^{*} \log (2)^{*}(\mathrm{f}-\mathrm{fD}) .^{\wedge} 2 / \mathrm{fwhm}{ }^{\wedge} 2\right)$;

psi $=$ ifft $\left(\mathrm{S} .^{*} \exp \left(\operatorname{sqrt}(-1) * 2^{*} \mathrm{pi}^{*}\right.\right.$ rand $\left.\left.(1, \mathrm{~N})\right)\right)$;

phi0 $=4^{*} \mathrm{pi}^{*} \mathrm{~L} 0 / \mathrm{lambda} 0+$ angle $(\mathrm{psi})$;

for $\mathrm{n}=1: \mathrm{N}$

$\mathrm{p}(\mathrm{n})=$ beta *abs(psi(n))* selmixpower $(\mathrm{C} 0 * \operatorname{abs}(\operatorname{psi}(\mathrm{n}))$, phi0 (n), alpha);

$\%$ Round-trip phase samples

$\%$ Generate the synthetic self-mixing signal end

$\%$ Feedback parameter, nominal value

$\%$ Linewidth enhancement factor

$\%$ Simulation time (s)

$\%$ Number of samples

$\%$ Laser power modulation coefficient

$\%$ Laser wavelength (m)

$\%$ Target nominal distance (m)

$\%$ Doppler signal FWHM $(\mathrm{Hz})$

$\%$ Doppler peak frequency $(\mathrm{Hz})$

$\%$ Noise (RMS value)

$\%$ Sample times

$\%$ Self-mixing signal samples

(Listing continued) 
noisy_signal $=\mathrm{p}+$ noise $/ 2 * \operatorname{randn}(1, \mathrm{~N})$;

$\%$ Add synthetic noise to the self-mixing

samples

fft_size $=256 ;$ [spectra, $\mathrm{f} 2]=$ pwelch (noisy_signal, fft_size, [], fft_size, N/T);

$\%$ PSD

plot (f2/1000, 10* $\log 10$ (spectra)); xlabel ('Frequency $(\mathrm{kHz})$ '); ylabel ('P.S.D. $(\mathrm{dBV} \wedge 2 / \mathrm{Hz}$ )');

This research was supported under the Australian Research Council's Discovery Projects funding scheme (DP 120 103703) and the European Cooperation in Science and Technology (COST) Action BM1205. Y. L. L. acknowledges support under the Queensland Government's Smart Futures Fellowships programme.

\section{References}

1. D. M. Kane and K. A. Shore, eds., Unlocking Dynamical Diversity: Optical Feedback Effects on Semiconductor Lasers (Wiley, 2005).

2. S. Donati, "Laser interferometry," in Electro-Optical Instrumentation (Prentice Hall, 2004), Chap. 4

3. S. Goeman, S. Boons, B. Dhoedt, K. Vandeputte, K Caekebeke, P. Van Daele, and R. Baets, "First demonstration of highly reflective and highly polarization selective diffraction gratings (GIRO-gratings) for long-wavelength VCSELs," IEEE Photon. Technol. Lett. 10, 1205-1207 (1998).

4. K. Petermann, "External optical feedback phenomena in semiconductor lasers," IEEE J. Sel. Top. Quantum Electron. 1, 480-489 (1995).

5. U. Fiedler and K. J. Ebeling, "Design of VCSEL's for feedback insensitive data transmission and external cavity active mode-locking," IEEE J. Sel. Top. Quantum Electron. 1, 442450 (1995).

6. G. Beheim and K. Fritsch, "Range finding using frequencymodulated laser diode," Appl. Opt. 25, 1439-1442 (1986).

7. T. Bosch, N. Servagent, and S. Donati, "Optical feedback interferometry for sensing application," Opt. Eng. 40, 20-27 (2001).

8. G. Giuliani, M. Norgia, S. Donati, and T. Bosch, "Laser diode self-mixing technique for sensing applications," J. Opt. A 4, S283-S294 (2002).

9. F. F. M. de Mul, M. H. Koelink, A. L. Weijers, J. Greve, J. G. Aarnoudse, R. Graaff, and A. C. M. Dassel, "Self-mixing laserDoppler velocimetry of liquid flow and of blood perfusion in tissue," Appl. Opt. 31, 5844-5851 (1992).

10. P. G. R. King and G. J. Steward, "Metrology with an optical maser," New Scientist 17, 180 (1963).

11. S. Donati, "Laser interferometry by induced modulation of cavity field," J. Appl. Phys. 49, 495-497 (1978).

12. J. H. Churnside, "Laser Doppler velocimetry by modulating a $\mathrm{CO}_{2}$ laser with backscattered light," Appl. Opt. 23, 61-66 (1984).

13. A. Valavanis, P. Dean, Y. L. Lim, R. Alhathlool, M. Nikolić, R. Kliese, S. P. Khanna, D. Indjin, S. J. Wilson, A. D. Rakić, E. H. Linfield, and A. G. Davies, "Self-mixing interferometry with terahertz quantum cascade lasers," IEEE Sensors J. 13, 37-43 (2013).

14. K. Bertling, Y. L. Lim, T. Taimre, D. Indjin, P. Dean, R. Weih, S. Höfling, M. Kamp, M. von Edlinger, J. Koeth, and A. D. Rakić, "Demonstration of the self-mixing effect in interband cascade lasers," Appl. Phys. Lett. 103, 231107 (2013).

15. Y. L. Lim, M. Nikolić, K. Bertling, R. Kliese, and A. D. Rakić, "Self-mixing imaging sensor using a monolithic VCSEL array with parallel readout," Opt. Express 17, 5517-5525 (2009).

16. P. Nerin, P. Puget, P. Besesty, and G. Chartier, "Self-mixing using a dual-polarisation Nd:YAG microchip laser," Electron. Lett. 33, 491-492 (1997).

17. S. Sudo, T. Ohtomo, Y. Takahashi, T. Oishi, and K. Otsuka, "Determination of velocity of self-mobile phytoplankton using a self-mixing thin-slice solid-state laser," Appl. Opt. 48, 4049-4055 (2009).
18. D. Han, M. Wang, and J. Zhou, "Self-mixing speckle in an erbium-doped fiber ring laser and its application to velocity sensing," IEEE Photon. Technol. Lett. 19, 1398-1400 (2007).

19. X. Dai, M. Wang, Y. Zhao, and J. Zhou, "Self-mixing interference in fiber ring laser and its application for vibration measurement," Opt. Express 17, 16543-16548 (2009).

20. R. Lang and K. Kobayashi, "External optical feedback effects on semiconductor injection laser properties," IEEE J. Quantum Electron. QE-16, 347-355 (1980).

21. R. Teysseyre, F. Bony, J. Perchoux, and T. Bosch, "Laser dynamics in sawtooth-like self-mixing signals," Opt. Lett. 37, 3771-3773 (2012).

22. X. Raoul, T. Bosch, G. Plantier, and N. Servagent, "A doublelaser diode onboard sensor for velocity measurements," IEEE Trans. Instrum. Meas. 53, 95-101 (2004).

23. G. Acket, D. Lenstra, A. den Boef, and B. Verbeek, "The influence of feedback intensity on longitudinal mode properties and optical noise in index-guided semiconductor lasers," IEEE J. Quantum Electron. QE-20, 1163-1169 (1984).

24. D. Lenstra, B. Verbeek, and A. Den Boef, "Coherence collapse in single-mode semiconductor lasers due to optical feedback," IEEE J. Quantum Electron. QE-21, 674-679 (1985).

25. J. Mork, B. Tromborg, and J. Mark, "Chaos in semiconductor lasers with optical feedback: theory and experiment," IEEE J. Quantum Electron. 28, 93-108 (1992).

26. J. Ohtsubo, H. Kumagai, and R. Shogenji, "Numerical study of doppler dynamics in self-mixing semiconductor lasers," IEEE Photon. Technol. Lett. 21, 742-744 (2009).

27. J. P. Toomey, D. M. Kane, M. W. Lee, and K. A. Shore, "Nonlinear dynamics of semiconductor lasers with feedback and modulation," Opt. Express 18, 16955-16972 (2010).

28. J. Ohtsubo, "Theory of optical feedback in semiconductor lasers," in Semiconductor Lasers: Stability, Instability and Chaos, 3rd ed. (Springer, 2013), Chap. 4.

29. P. Spencer, P. Rees, and I. Pierce, "Theoretical analysis," in Unlocking Dynamical Diversity: Optical Feedback Effects on Semiconductor Lasers, D. M. Kane and K. A. Shore, eds. (Wiley, 2005), Chap. 2.

30. K. Petermann, "Semiconductor lasers with optical feedback," in Laser Diode Modulation and Noise (Kluwer Academic, 1988), Chap. 9.

31. W. M. Wang, W. J. O. Boyle, K. T. V. Grattan, and A. W. Palmer, "Self-mixing interference in a diode laser: experimental observations and theoretical analysis," Appl. Opt. 32, 1551-1558 (1993).

32. J. Perchoux and T. Bosch, "Multimode VCSELs for self-mixing velocity measurements," in IEEE Sensors (IEEE, 2007), pp. 419-422.

33. H. Jentink, F. F. M. de Mul, H. Suichies, J. Aarnoudse, and J. Greve, "Small laser Doppler velocimeter based on the selfmixing effect in a diode laser," Appl. Opt. 27, 379-385 (1988).

34. J. R. Tucker, A. D. Rakić, C. J. O'Brien, and A. V. Zvyagin, "Effect of multiple transverse modes in self-mixing sensors based on vertical-cavity surface-emitting lasers," Appl. Opt. 46, 611-619 (2007).

35. S. Imran, M. Yamada, and Y. Kuwamura, "Theoretical analysis of the optical feedback noise based on multimode model of semiconductor lasers," IEEE J. Quantum Electron. 48, 521-527 (2012).

36. J. Xi, Y. Yu, J. F. Chicharo, and T. Bosch, "Estimating the parameters of semiconductor lasers based on weak optical feedback self-mixing interferometry," IEEE J. Quantum Electron. 41, 1058-1064 (2005).

37. U. Zabit, F. Bony, T. Bosch, and A. D. Rakić, "A self-mixing displacement sensor with fringe-loss compensation for harmonic 
vibrations," IEEE Photon. Technol. Lett. 22, 410-412 (2010).

38. K. Bertling, J. R. Tucker, and A. D. Rakić, "Optimum injection current waveform for a laser range finder based on the selfmixing effect," Proc. SPIE 5277, 334-345 (2004).

39. G. Plantier, C. Bes, and T. Bosch, "Behavioral model of a selfmixing laser diode sensor," IEEE J. Quantum Electron. 41, 1157-1167 (2005).

40. L. A. Coldren and S. W. Corzine, "A phenomenological approach to diode lasers," in Diode Lasers and Photonic Integrated Circuits, K. Chang, ed. (Wiley, 1995), Chap. 2.

41. Y. Mitsuhashi, J. Shimada, and S. Mitsutsuka, "Voltage change across the self-coupled semiconductor laser," IEEE J. Quantum Electron. QE-17, 1216-1225 (1981).

42. S. Donati, "Responsivity and noise of self-mixing photodetection schemes," IEEE J. Quantum Electron. 47, 1428-1433 (2011).

43. T. Taimre and A. D. Rakić, "On the nature of Acket's characteristic parameter $C$ in semiconductor lasers," Appl. Opt. 53, 1001-1006 (2014).

44. L. A. Coldren and S. W. Corzine, Diode Lasers and Photonic Integrated Circuits (Wiley, 1995).

45. S. Shinohara, A. Mochizuki, H. Yoshida, and M. Sumi, "Laser Doppler velocimeter using the self-mixing effect of a semiconductor laser diode," Appl. Opt. 25, 1417-1419 (1986).

46. U. Zabit, O. Bernal, and T. Bosch, "Self-mixing laser sensor for large displacements: Signal recovery in the presence of speckle," IEEE Sensors J. 13, 824-831 (2013).

47. G. Giuliani and S. Donati, "Laser interferometry," in Unlocking Dynamical Diversity: Optical Feedback Effects on Semiconductor Lasers, D. M. Kane and K. A. Shore, eds. (Wiley, 2005), Chap. 7.

48. MathWorks, "fzero," http://www.mathworks.com/access/ helpdesk/help/techdoc/ref/fzero.html.
49. Y. L. Lim, P. Dean, M. Nikolić, R. Kliese, S. P. Khanna, M. Lachab, A. Valavanis, D. Indjin, Z. Ikonić, P. Harrison, E. H. Linfield, A. G. Davies, S. J. Wilson, and A. D. Rakić, "Demonstration of a self-mixing displacement sensor based on terahertz quantum cascade lasers," Appl. Phys. Lett. 99, 081108 (2011).

50. F. Gouaux, N. Servagent, and T. Bosch, "Absolute distance measurement with an optical feedback interferometer," Appl. Opt. 37, 6684-6689 (1998).

51. A. D. Rakić, T. Taimre, K. Bertling, Y. L. Lim, P. Dean, D. Indjin, Z. Ikonić, P. Harrison, A. Valavanis, S. P. Khanna, M. Lachab, S. J. Wilson, E. H. Linfield, and A. G. Davies, "Swept-frequency feedback interferometry using terahertz frequency QCLs: a method for imaging and materials analysis," Opt. Express 21, 22194-22205 (2013).

52. P. J. de Groot and G. M. Gallatin, "Three-dimensional imaging coherent laser radar array," Opt. Eng. 28, 284456 (1989).

53. R. Kliese and A. D. Rakić, "Spectral broadening caused by dynamic speckle in self-mixing velocimetry sensors," Opt. Express 20, 18757-18771 (2012).

54. D. P. Kroese, T. Taimre, and Z. I. Botev, "Random process generation," in Handbook of Monte Carlo Methods (Wiley, 2011), Chap. 5.

55. K. Y. R. Billah and M. Shinozuka, "Numerical method for colored-noise generation and its application to a bistable system," Phys. Rev. A 42, 7492-7495 (1990).

56. D. J. Young and N. C. Beaulieu, "The generation of correlated Rayleigh random variates by inverse discrete Fourier transform," IEEE Trans. Commun. 48, 1114-1127 (2000).

57. P. Welch, "The use of fast Fourier transform for the estimation of power spectra: A method based on time averaging over short, modified periodograms," IEEE Trans. Audio Electroacoust. 15, 70-73 (1967). 